\title{
Análise do teor de cocaína em amostras apreendidas pela polícia utilizando-se a técnica de cromatografia liquida de alta eficiência com detector UV-Vis
}

\author{
Marcelo Firmino de Oliveira *1, Jacqueline Querino Alves ${ }^{1}$, José Fernando de Andrade ${ }^{1}$, Adelir Aparecida \\ Saczk², Leonardo Luiz Okumura ${ }^{3}$ \\ 1 - Departamento de Química, Faculdade de Filosofia, Ciências e Letras de Ribeirão Preto, Universidade de São Paulo. Av. Bandeirantes \\ 3900, CEP 14040-901, Ribeirão Preto - SP, Brasil. \\ 2 - Departamento de Química, Universidade Federal de Lavras. Campus Universitário, Caixa Postal 3037, CEP 37200-000, Lavras - \\ $M G$, Brasil. \\ 3 - Departamento de Química, Centro de Ciências Exatas, Universidade Federal de Viçosa. Av. Peter Henry Rolfs, s/n, CEP 36570-000, \\ Viçosa-MG, Brasil. \\ *email:marcelex@ffclrp.usp.br
}

\begin{abstract}
Resumo: A técnica de CLAE com detecção UV-Vis foi empregada na análise do teor de cocaína em amostras apreendidas de cocaína e crack. Uma fase móvel de acetonitrila/água (95:5v/v) possibilitou um sinal de pico para a cocaína em 3,5 minutos. O sinal espectrofotométrico otimizado foi obtido em um comprimento de onda de $224 \mathrm{~nm}$. A curva analítica de 1,0 a 40,0 ppm para cocaína foi obtida, exibindo um coeficiente de correlação linear de 0,9989, com limites de detecção e quantificação de 0,75 e $3,78 \mathrm{ppm}$, respectivamente. Esta metodologia foi aplicada na dosagem de amostras confiscadas de cocaína e crack no Laboratório de Polícia Científica de Ribeirão Preto-SP.
\end{abstract}

Palavras-chave: cocaína, CLAE, química forense

\section{1 - INTRODUÇÃO}

\section{A problemática das drogas de abuso}

Um dos problemas que mais afetam o contexto social atual é o consumo de drogas ilícitas. Isso porque este consumo reflete não só na saúde, no convívio familiar e social, como também movimenta milhões por ano, consistindo em um mercado financeiro ilícito não só no Brasil, mas em muitos outros países também [1-3]. Também há que se considerar que indivíduos usuários de drogas certamente são mais suscetíveis à prática de crimes, tanto devido ao fato de geralmente ficarem racionalmente afetados quando do uso da droga, quanto por necessidade de angariar fundos para a mantença do vício. Faz jus ao destaque uma das atuais medidas legislativas tomadas com relação ao assunto supra, a lei 11.343 de 23 de agosto de 2006 [4], a qual instituiu o SISNAD - Sistema Nacional de Políticas Públicas sobre Drogas - e dispõe não somente sobre os crimes relacionados às drogas, mas também aponta medidas relativas à prevenção e reinserção social de usuários e dependentes de substâncias desta classe [2].

E, não obstante opiniões contrárias, a posse de pequena quantidade já configura o delito previsto no artigo 28 da Lei $11.343 / 06$, o que comprova serem tais substâncias realmente nocivas à saúde humana, bem como à sociedade em si mesma considerada. Várias são as medidas que podem ser tomadas visando o combate ao uso e tráfico 
de drogas e, nestas, certamente, tem papel de merecido destaque, o incentivo à pesquisa em química forense e subseqüente aplicação destes conhecimentos nos laboratórios de polícia científica. Com relação ao Estado de São Paulo, os dados referentes às apreensões no ano de 2008 (total de 24.930), em comparação a 2007 (total de 23.127), apresentam aumento de 6,9\%. Considerando-se desde 1999 (total de 10.525), houve um acréscimo de $105,6 \%$ nas apreensões [3].

\section{Cocaina e crack}

A cocaína constitui o mais importante alcalóide natural extraído das folhas do arbusto Erythroxylon coca [5] sendo sua estrutura reportada na Figura 1. Em sua forma purificada, esta espécie constitui um sólido branco, cristalino, de odor aromático, sob a forma de um sal, o cloridrato de cocaína, o qual é solúvel em água e, portanto, pode ser aspirado ou dissolvido em água para uso endovenoso. Adicionalmente, uma nova forma de consumo da cocaína, constitui na mistura da base livre da cocaína com bicarbonato de sódio, tornando a mistura alcalinizada, originando uma massa petrificada, geralmente de aspecto marromamarelado, popularmente conhecida como "crack", o qual é pouco solúvel em água, mas que se volatiliza facilmente quando aquecido $\left(95^{\circ} \mathrm{C}\right)$, é comumente fumado em cachimbos improvisados, conhecidos por "maricas". A palavra "crack" é derivada do ruído característico que é produzido pelas pedras quando estão sendo decompostas pelo fumo. Os efeitos iniciais da cocaína no organismo constituem em euforia, excitação, insônia e supressão do apetite, devido à ação no sistema nervoso central [4].

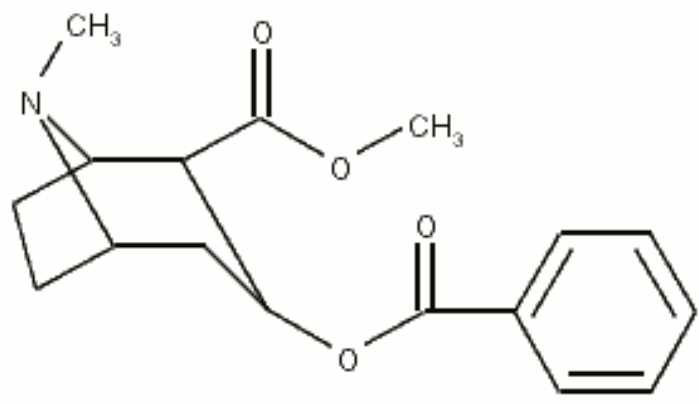

Figura 1: Estrutura molecular da cocaína.
A análise de cocaína pelas técnicas de cromatografia líquida de alta eficiência (CLAE) [69] e cromatografia gasosa (CG) [10-16] tem sido amplamente reportada na literatura, indicando o potencial das mesmas para as análises forenses. A boa acuracidade e robustez, aliadas à possibilidade de obtenção de limites de detecção na ordem de $n g \mathrm{~mL}^{-1}$ para a cocaína utilizando-se tais metodologias, possibilitou a aplicação destas técnicas cromatográficas na análise do referido entorpecente em diversas matrizes, tais como fluidos biológicos $[7,15,16]$, cabelo $[6,8,14]$ e peças de interesse forense, como papel moeda [16], indicando a versatilidade de tais métodos. Neste sentido, este trabalho visa investigar a aplicabilidade da técnica de CLAE com detector UV-Vis na análise de rotina de cocaína em amostras de cocaína e crack apreendidas pela polícia.

\section{2 - PARTE EXPERIMENTAL}

\section{Materiais e reagentes}

As amostras de cocaína e crack, bem como os padrões purificados de cocaína foram fornecidos pelo Núcleo de Exames Toxicológicos - Núcleo de Perícias Criminalísticas de Ribeirão Preto-SP. Todas as amostras analisadas apresentaram resultado positivo para testes prévios colorimétricos para cocaína. Adicionalmente, foram utilizadas amostras de processos judiciais concluídos. O padrão cromatográfico de cocaína utilizado foi uma solução de cocaína (base livre) em acetonitrila na dosagem de $1000 \mathrm{ppm}$, da Cerrilliant.

O solvente orgânico utilizado na fase móvel cromatográfica foi acetonitrila da Merck, padrão HPLC. Para a composição aquosa desta fase móvel, utilizou-se água deionizada.

\section{Preparo das soluções-padrão de cocaína}

O preparo das soluções-padrão foi efetuado por diluição de solução padrão 1000 ppm, em diferentes volumes finais de acetonitrila, sendo obtidos os padrões de $1,0,5,0,10,0 \quad 20,0$ e 40,0 ppm. 


\section{Preparo das amostras}

Visando-se obter uma metodologia rápida e prática de análise, cada alíquota de $2,5 \mathrm{mg}$ de amostra foi dissolvida diretamente em $100 \mathrm{~g}$ de acetonitrila, estando portanto em $25 \mathrm{ppm}$ de amostra.

\section{Dispositivo cromatográfico}

Foi utilizado um equipamento de CLAE da marca Shimadzu, com controle de equipamento e aquisição de dados por intermédio de um microcomputador acoplado. O fluxo de solução de fase móvel foi mantido em condições isocráticas, a 1,0 $\mathrm{mL}$ por minuto, com fase móvel de acetonitrila e água, na proporção de 95:5 v/v. A válvula de injeção foi composta por alça de amostragem de 20 $\mu \mathrm{L}$. Fez-se uso de uma coluna $\mathrm{C}_{18}$ de $15 \mathrm{~cm}$ da Labtron acompanhada da coluna de guarda também $\mathrm{C}_{18}$, de mesma marca. O comprimento de onda foi selecionado com base no máximo de absorbância obtida para a cocaína. As soluções de amostras de cocaína foram injetadas diretamente no cromatógrafo, sendo realizada tão somente uma filtração prévia por seringa dotada de filtro descartável.

\section{Análise quantitativa}

A curva analítica para dosagem de cocaína foi obtida por regressão linear, plotando-se as áreas dos picos obtidos para cada solução-padrão de cocaína em função das concentrações. O teor de cocaína nas amostras desconhecidas de cocaína e crack foi obtido pela integração das áreas dos picos pertinentes à cocaína nos cromatogramas das amostras e subseqüente extrapolação da área obtida na curva analítica. A análise das soluções de padrões, bem como das soluções de amostras foi feita em triplicata.

\section{3 - RESULTADOS E DISCUSSÃO}

\section{Seleção da fase móvel}

Para as medições cromatográficas efetuadas neste trabalho, foi escolhida a mistura de acetonitrila e água, na proporção 95:5 v/v como fase móvel, em decorrência das facilidades encontradas quando do uso desse sistema, em testes prévios realizados neste projeto. Valores otimizados de tempo de retenção abaixo de 5 minutos, bem como picos bem definidos são obtidos quando acetonitrila é empregada.

\section{Seleção do comprimento de onda}

Os valores de absorbância foram medidos em comprimento de onda igual a $224 \mathrm{~nm}$, dado ser este o comprimento de onda com máximo de absorção encontrado para a molécula de cocaína neste experimento.

\section{Análise de cocaína em amostras por CLAE}

Os cromatogramas obtidos para a cocaína em diferentes concentrações no sistema cromatográfico proposto indicaram um tempo de retenção ao redor de 3,5 minutos, conforme reportado na Figura 2. A curva analítica obtida para a cocaína no intervalo de 1,0 a 40,0 ppm (Figura 2) apresentou um coeficiente de correlação linear de 0,9989 e uma sensibilidade espectrofotométrica de 87,5 $\mathrm{ppm}^{-1}$. Considerando-se o desvio padrão obtido para a referida curva em 21,9 , foi possível calcular os limites de detecção e quantificação para a cocaína em 0,75 ppm e 3,78 ppm, respectivamente, utilizando-se as relações $3 \mathrm{SD} / \mathrm{m}$ e $10 \mathrm{SD} / \mathrm{m}$ ( $\mathrm{SD}=$ desvio padrão da curva; $\mathrm{m}=$ sensibilidade da curva analítica). 


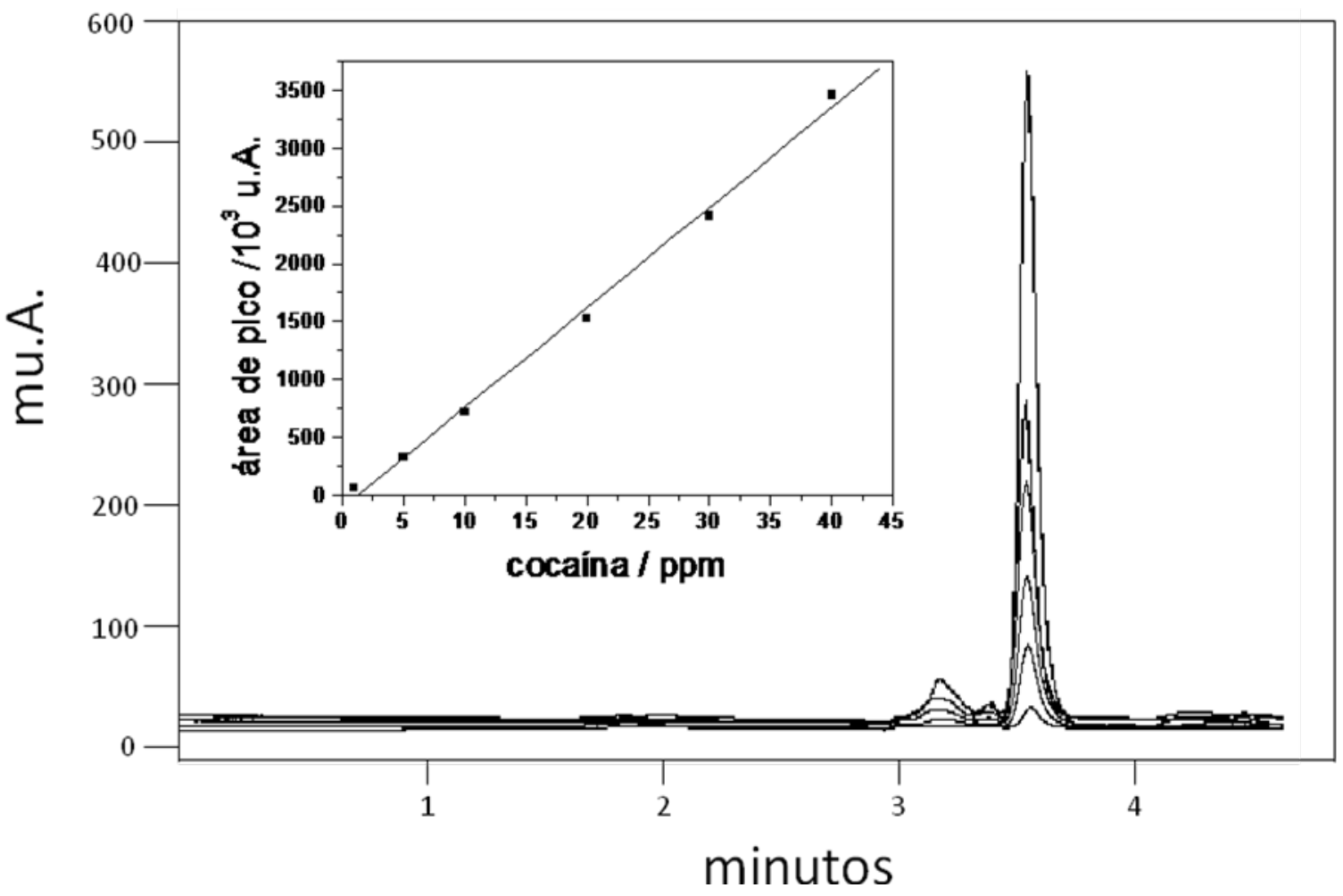

Figura 2: cromatogramas obtidos para diferentes concentrações de cocaína: 1,0 , 5,0 , 10,0 , 20,0, 30,0 e 40,0ppm. Fase móvel: acetonitrila:água (95:5); vazão: $1,0 \mathrm{~mL} / \mathrm{min}$; deteç̧ão em $224 \mathrm{~nm}$.

Os estudos de recuperação para a técnica proposta situaram-se em um intervalo entre 95\% e $101 \%$, com coeficientes de variação (CV) entre $0,4 \%$ e 3,9\%, indicando uma elavada precisão na analise de cocaína, tanto na forma cloridrato quanto na forma de base livre (crack). A presença de substâncias contaminantes e adulterantes nas amostras estudadas não constituiu em interferência para tais análises, uma vez que as mesmas apresentam diferentes tempos de retenção na eluição da fase móvel, conforme evidenciado em um típico cromatograma de solução de amostra apreendida de crack, na Figura 3.

Considerando-se um período de 6 minutos para uma análise completa da solução de amostra de cocaína sem efeitos de carryover, é possível estimar uma freqüência analítica de 10 análises por hora, ou 80 análises por dia, considerando-se somente período comercial de 8 horas diárias. Neste contexto, tal metodologia atende perfeitamente a demanda local para estas análises, conforme informações coletadas no laboratório de polícia científica local. 


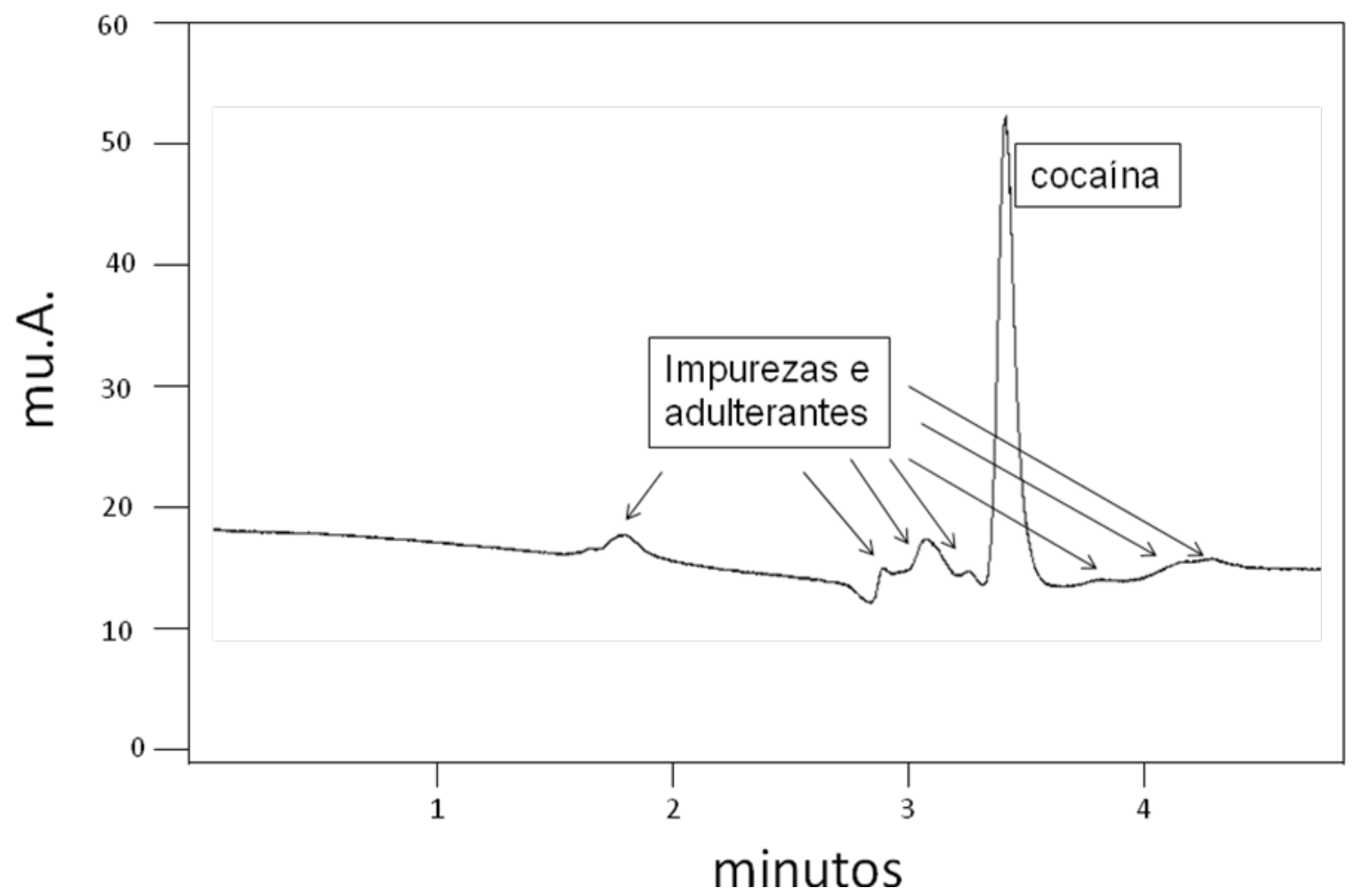

Figura 3: Cromatograma típico obtido para solução de amostra apreendida de cocaína, atentando-se para a presença de substâncias contaminantes/adulterantes na composição da mesma. Fase móvel: acetonitrila:água (95:5); vazão: 1,0mL/min; detecção em $224 \mathrm{~nm}$.

Utilizando-se a curva analítica obtida, bem como os cromatogramas obtidos para cada solução de amostra, foi possível calcular os teores de cocaína nas amostras de cocaína e crack estudadas neste trabalho, sendo estes resultados reportados na Tabela 1, em ordem decrescente de teor de cocaína. 
Tabela 1: Resultados obtidos para a dosagem de cocaína em amostras apreendidas pela polícia utilizando-se a técnica CLAE com detector UV-Vis ( $\mathrm{SD}=$ desvio padrão para 3 medidas; CV = coeficiente de variação).

\begin{tabular}{cccccc}
\hline Amostra & $\begin{array}{c}\text { [cocaína] } \\
\text { esperada (ppm) }\end{array}$ & $\begin{array}{c}\text { [cocaína] } \\
\text { obtida (ppm) }\end{array}$ & SD & CV(\%) & Dosagem (\%) \\
\hline 1 & 25,0 & 23,9 & 0,9 & 3,7 & 95,6 \\
2 & 25,0 & 21,5 & 0,5 & 2,4 & 86,0 \\
3 & 25,0 & 19,4 & 0,2 & 1,2 & 77,6 \\
4 & 25,0 & 16,4 & 0,3 & 1,9 & 65,6 \\
5 & 25,0 & 16,2 & 0,1 & 0,7 & 64,9 \\
6 & 25,0 & 9,4 & 0,1 & 1,3 & 37,4 \\
7 & 25,0 & 5,4 & 0,2 & 3,2 & 21,6 \\
9 & 25,0 & 4,7 & 0,1 & 2,9 & 18,9 \\
10 & 25,0 & 4,4 & 0,2 & 3,9 & 17,5 \\
\hline
\end{tabular}

Os resultados obtidos indicaram a presença de cocaína em todas as amostras analisadas, com teores compreendidos entre $37,4 \%$ e $95,6 \%$ para amostras de cocaína e entre $13,6 \%$ e 21,6\% para amostras de crack. Estes resultados estão coerentes com o perfil químico observado para estas espécies, ou seja, um menor teor de cocaína nas amostras de crack é esperado, uma vez que a produção desta forma de entorpecente decorre do reaproveitamento das sobras das etapas de síntese da cocaína cloridrato.

\section{4 - CONCLUSÕES}

A técnica cromatográfica proposta neste trabalho para a análise do teor de cocaína em amostras apreendidas apresentou boa repetibilidade e acuracidade. A freqüência analítica de 80 análises/dia viabiliza a referida técnica para ser aplicada em análises de rotina em laboratórios forenses, tanto na análise de amostras de cocaína quanto de crack.

Esta técnica instrumental apresenta inúmeras vantagens em relação aos testes clássicos convencionais colorimétricos empregados em análises de rotina, tais como a possibilidade de quantificação das amostras, bem como a eliminação de possibilidades de resultados do tipo falso positivo, inerentes aos exames colorimétricos, oferecendo desta forma, informações cruciais na etapa de investigação policial. Como exemplo, pode-se obter um perfil químico das drogas apreendidas, com base na deteç̧ão tanto da droga quanto dos interferentes que a constituem, podendo-se corre- lacionar as amostras apreendidas a determinados traficantes ou determinadas regiões do planeta. De acordo com a literatura, esta é a primeira vez que um estudo sobre dosagem de cocaína em amostras apreendidas de entorpecentes pela técnica de CLAE é reportado no Brasil.

Analysis of cocaine content in samples apprehended by Police using the high-performance liquid chromatography technique with UV-Vis detector. 
Abstract: The HPLC technique with UV-Vis detection was employed in the analysis of cocaine content in apprehended samples of cocaine and crack. A peak signal for cocaine was obtained in 3.5 minutes run by using acetonitrile/water $(95: 5 \mathrm{v} / \mathrm{v})$ as a mobile phase. Optimized spectrophotometric signal was obtained at a wavelength of $224 \mathrm{~nm}$. The analytical curve from 1.0 to $40.0 \mathrm{ppm}$ of cocaine was obtained, showing a linear correlation coefficient of 0.9989 , with detection and quantification limits of $0.75 \mathrm{ppm}$ and $3.78 \mathrm{ppm}$, respectively. This methodology was employed at the dosage of confiscated samples of cocaine and crack in the Scientific Police Laboratory of Ribeirão Preto-SP city.

Keywords: cocaine, HPLC, forensic chemistry

\section{5 - AGRADECIMENTOS}

Os autores agradecem o suporte financeiro da Fundação de Amparo à Pesquisa do Estado de São Paulo (FAPESP) e do Conselho Nacional de Desenvolvimento Científico e Tecnológico (CNPq).

\section{REFERÊNCIAS}

[1] - Santos, M. J.; Kassouf, A.L. Economia, 8 (2007) 187. [2] - Fajnzylber, P., Lederman, D., \& Loayza, N. Determinants of Crime Rates in Latin America and the World: Viewpoints. The World Bank, Washington, 1998.

[3] - Coordenadoria de Análise e Planejamento. Secretaria da Segurança Pública. Estudos Criminológicos 3 . Disponível:

$<$ http://www.ssp.sp.gov.br/estatisticas/downloads/manual_estudos_criminologicos_3.pdf>. Acesso: 28 abr. 2009, 14:19:00.

[4] - Lei 11.343/ 2006 Em: Vade Mecum. Obra Coletiva de autoria da Editora Saraiva com a colaboração de Antonio Luiz Toledo, Márcia Cristina Vaz dos Santos Windt e Lívia Céspedes. 7. ed. atual. ampl. Saraiva: São Paulo, 2009. p. 1731.

[5] - J. L. Zarzuela, Química Legal. Em: Tochetto, D. et al. Tratado de Perícias Criminalísticas. ed. Sagra-DC Luzzatto, Porto Alegre, 1995, cap. 8, p. 164-169.

[6] - M. L. Menezes, G. A. Muzardo, M. S. Chaves, J. Liq. Chrom. Rel. Tech., 27 (2004) 1799.

[7] - P. Fernandez, L. Morales, Forensic Sci. Int. 161 (2006) 31.

[8] - F. Tagliaro, R. Valentini et al., Forensic Sci. Int. 107 ( 2000) 121.

[9] - P. Fernandez, M. Leon et al., J. Liq. Chrom. Rel. Tech. 26 ( 2003) 2003.

[10] - F. S. Romolo, M. Rotolo, et al., Forensic Sci. Int. 138 (2003) 17.

[11] - M. Montagna, A. Polentini et al. Forensic Sci. Int. 128 (2002) 79 .
[12] - L. Skender, V. Karacic, et al., Forensic Sci. Int. 125 (2002) 120.

[13] - O. Quintela, A. M. Bermejo et al., Forensic Sci. Int. 107 (2000) 273.

[14] - M. C. Ricossa, M. Bernini, F. De Ferrari, Forensic Sci. Int., 107 (2000) 301.

[15] - L. Bujan, P. Fernandez, Anal. Lett., 34 (2001) 2263.

[16] -E. Donato, C.C.S. Martin, B. S. Martinis, Quim. Nova 30 (2007) 1966. 